\title{
THE COMMON CRYOGENIC TEST FACILITY FOR THE ATLAS BARREL AND END-CAP TOROID MAGNET
}

\author{
N. Delruelle, F. Haug, S. Junker, G. Passardi, R. Pengo and O. Pirotte
}

The large ATLAS toroidal superconducting magnet made of the Barrel and two End-Caps needs extensive testing at the surface of the individual components prior to their final assembly into the underground cavern of LHC. A cryogenic test facility specifically designed for cooling sequentially the eight coils making the Barrel Toroid (BT) has been fully commissioned and is now ready for final acceptance of these magnets. This facility, originally designed for testing individually the 46 tons BT coils, will be upgraded to allow the acceptance tests of the two End-Caps, each of them having a 160 tons cold mass. The integrated system mainly comprises a $1.2 \mathrm{~kW} @ 4.5 \mathrm{~K}$ refrigerator, a $10 \mathrm{~kW}$ liquid-nitrogen precooler, two cryostats housing liquid helium centrifugal pumps of respectively $80 \mathrm{~g} / \mathrm{s}$ and $600 \mathrm{~g} / \mathrm{s}$ nominal flow and specific instrumentation to measure the thermal performances of the magnets. This paper describes the overall facility with particular emphasis to the cryogenic features adopted to match the specific requirements of the magnets in the various operating scenarios.

Accelerator Technology Department

\author{
Presented at the 2003 Cryogenic Engineering Conference and International Cryogenic Materials Conference \\ CEC/ICMC 2003 \\ 22-26 September 2003, Anchorage, Alaska
}

Administrative Secretariat

AT Division

CERN

CH - 1211 Geneva 23
Geneva, Switzerland

29 January 2004 


\title{
THE COMMON CRYOGENIC TEST FACILITY FOR THE ATLAS BARREL AND END-CAP TOROID MAGNETS
}

\author{
N. Delruelle, F. Haug, S. Junker, G. Passardi, R. Pengo and O. Pirotte \\ CERN, AT division, 1211 Geneva 23, Switzerland
}

\begin{abstract}
The large ATLAS toroidal superconducting magnet made of the Barrel and two EndCaps needs extensive testing at the surface of the individual components prior to their final assembly into the underground cavern of LHC. A cryogenic test facility specifically designed for cooling sequentially the eight coils making the Barrel Toroid (BT) has been fully commissioned and is now ready for final acceptance of these magnets. This facility, originally designed for testing individually the 46 tons BT coils, will be upgraded to allow the acceptance tests of the two End-Caps, each of them having a 160 tons cold mass. The integrated system mainly comprises a $1.2 \mathrm{~kW} @ 4.5 \mathrm{~K}$ refrigerator, a $10 \mathrm{~kW}$ liquid-nitrogen precooler, two cryostats housing liquid helium centrifugal pumps of respectively $80 \mathrm{~g} / \mathrm{s}$ and $600 \mathrm{~g} / \mathrm{s}$ nominal flow and specific instrumentation to measure the thermal performances of the magnets. This paper describes the overall facility with particular emphasis to the cryogenic features adopted to match the specific requirements of the magnets in the various operating scenarios.
\end{abstract}

\section{INTRODUCTION}

The ATLAS detector is the largest experiment of the Large Hadron Collider (LHC) and will be installed $100 \mathrm{~m}$ underground in a $50000 \mathrm{~m}^{3}$ cavern located at collision point 1 . The ATLAS magnet system is based on a thin superconducting Central Solenoid (CS) which generates a uniform longitudinal field for the inner tracker, and three superconducting toroid magnets (a Barrel and two End-Caps) producing a tangential field around the central axis for the muon spectrometer.

The CS is $5.3 \mathrm{~m}$ in length with an inner diameter of $2.5 \mathrm{~m}$ and has a cold mass of 5.4 tons. The Barrel Toroid (BT), surrounding the CS, is made of 8 superconducting coils symmetrically placed around the beam axis of the detector. The BT has an axial length of $25.3 \mathrm{~m}$, an outer diameter of $20.1 \mathrm{~m}$, a stored energy of $1080 \mathrm{MJ}$ and a total mass of 830 tons with a cold mass of 370 tons. Two identical End-Caps Toroids (ECT), each of them also made of 8 coils, are inserted at the two ends of the BT in order to provide the 
required magnetic field configuration in the forward areas of the detector. The inner bore of an ECT has a diameter of $1.7 \mathrm{~m}$, the outer diameter is $10.7 \mathrm{~m}$ and its axial length is $5 \mathrm{~m}$. The stored energy of each ECT is $250 \mathrm{MJ}$, its total mass is 239 tons of which 160 tons are the cold mass.

Because of the exceptional dimensions, it was not feasible to assemble and test on the surface the entire BT and then move it as a whole to its final underground area. Therefore, it was decided to test individually at the surface the eight coils forming the BT and the ECTs in two dedicated facilities providing, as closely as possible, the real operating conditions from both magnetic and cryogenic points of view.

\section{CRYOGENIC TEST FACILITY CONCEPT}

The BT cryogenic test station was designed and set-up with a helium distribution system allowing three test benches, with two of them holding one of the eight rectangular coils forming the BT [1]. This facility has been successfully commissioned with prototype magnets in 2001 [2]. After a subsequent decision of the ATLAS Collaboration to test also the ECTs in the same area (Fig. 1), it became necessary to substantially upgrade the capabilities of the BT test station.

In fact, a thermal analysis of the entire facility showed that the cooling capacity of the existing refrigerator $(1.2 \mathrm{~kW} @ 4.5 \mathrm{~K})$ was not sufficient to cope with the higher ECT thermal loads. Table 1 summarizes the main thermal characteristics of the ATLAS toroids and indicates that for a single ECT, thermal loads on both $4.5 \mathrm{~K}$ and shield circuits are almost three times larger than for a single BT.

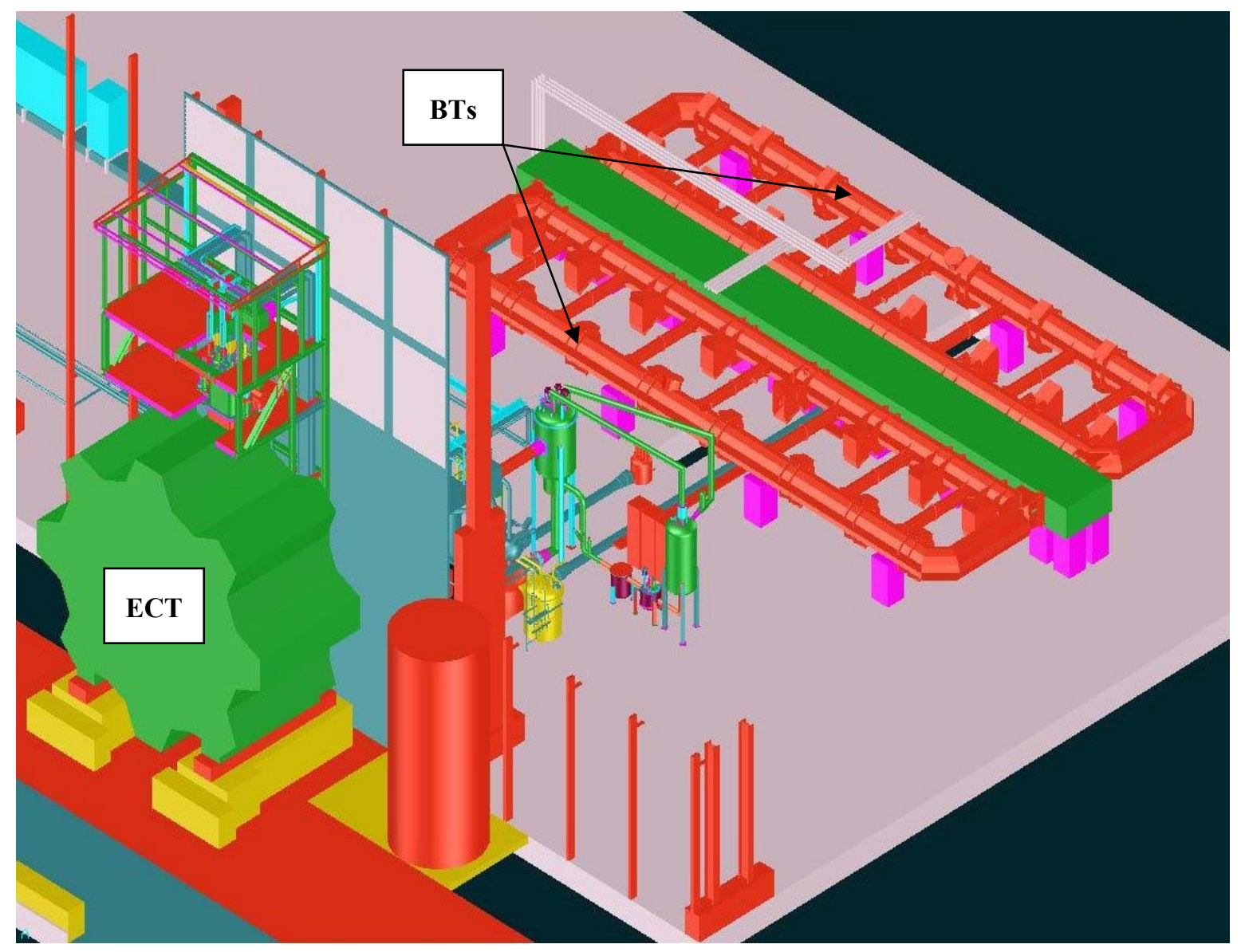

FIGURE 1: Three-dimensional view of the test facility with two BTs and one ECT installed. 
TABLE 1: Thermal loads and main characteristics of the ATLAS toroid magnets.

\begin{tabular}{lcc}
\hline & For a single BT & For a single ECT \\
\hline Static isothermal load at 4.5 K, with an average shield & & \\
temperature $\approx 0 \mathrm{~K}$ & $80 \mathrm{~W}$ & $180 \mathrm{~W}$ \\
Dynamic isothermal load at 4.5 K (current ramp up/down) & $44 \mathrm{~W}$ & $110 \mathrm{~W}$ \\
Liquefaction load at 4.5 K (current leads) & $3.1 \mathrm{~g} / \mathrm{s}$ & $3.1 \mathrm{~g} / \mathrm{s}$ \\
Thermal shield load between 40 K and 80 K & $740 \mathrm{~W}$ & $2250 \mathrm{~W}$ \\
Pump-flow required at 4.5 K during surface test & $80 \mathrm{~g} / \mathrm{s}$ & $300 \mathrm{~g} / \mathrm{s}$ \\
Precooler helium flow required during cool-down in surface test & $50 \mathrm{~g} / \mathrm{s}$ & $50 \mathrm{~g} / \mathrm{s}$ \\
Total assembly mass & $104 \mathrm{tons}$ & 239 tons \\
Cold mass & 46 tons & 160 tons \\
\hline
\end{tabular}

The third test bench will be used for the ECTs. The corresponding simplified cooling scheme is shown in Fig. 2 and the following solutions will be adopted in order to boost the overall performance of the existing facility.

The concept adopted for the BT tests, i.e. to cool-down the magnet from $300 \mathrm{~K}$ to $100 \mathrm{~K}$ with a $10 \mathrm{~kW}$ liquid-nitrogen $\left(\mathrm{LN}_{2}\right)$ precooler and then to switch off this precooler and continue the cooling with solely the $1.2 \mathrm{~kW}$ refrigerator down to the operating conditions is abandoned for the ECT and we keep on duty the $\mathrm{LN}_{2}$ precooler, even when the ECT temperature is below $100 \mathrm{~K}$. With this concept, the $1.2 \mathrm{~kW}$ refrigerator provides the $4.5 \mathrm{~K}$ and current leads cooling while the precooler is coping with the ECT thermal

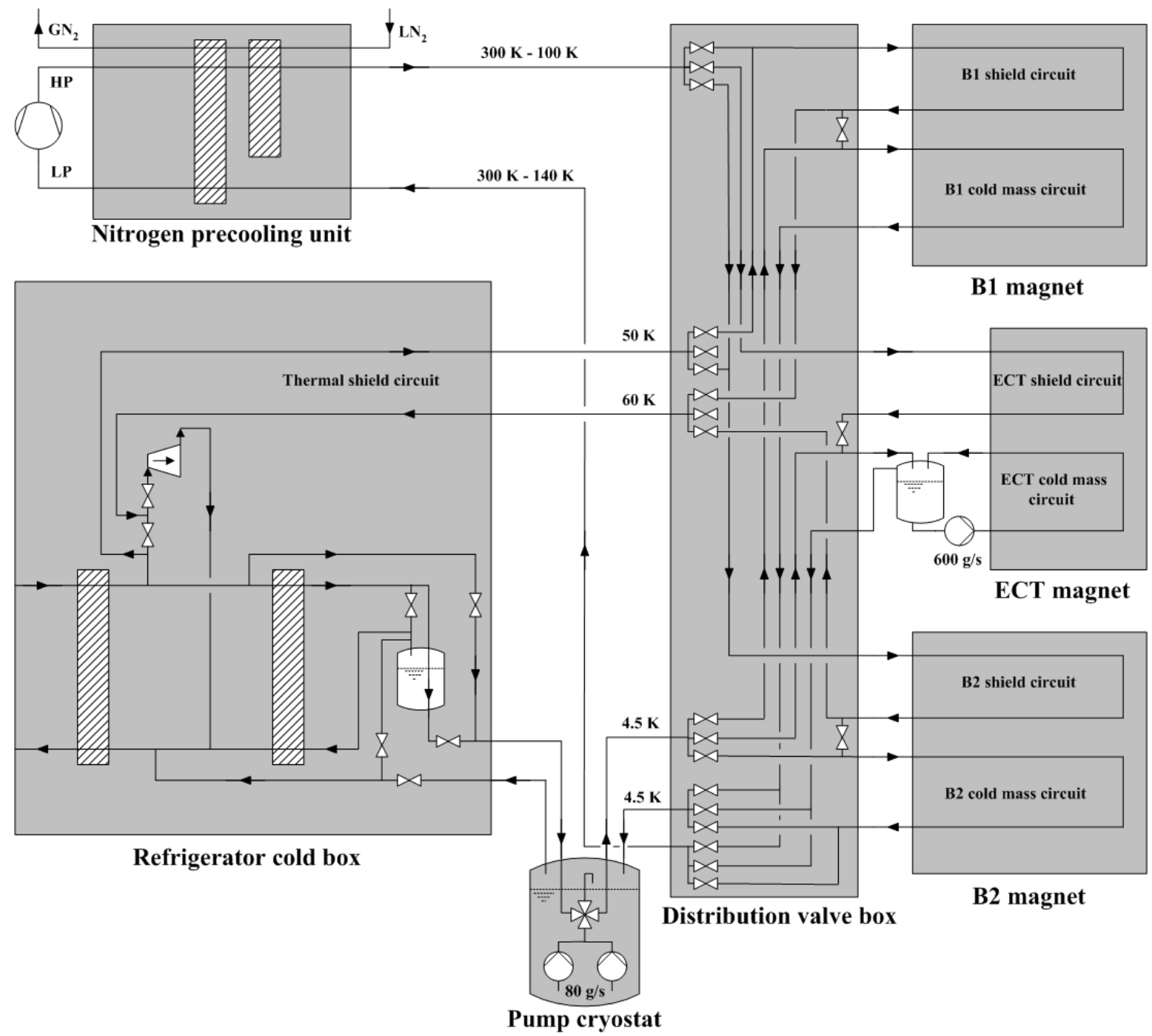

FIGURE 2: Simplified Process Flow Diagram of the cryogenic test station. 
shield load. Furthermore, we will use $\mathrm{LN}_{2}$ in the first heat exchanger of the $1.2 \mathrm{~kW}$ cold box to increase its liquefaction capacity. A drawback of this solution is that the ECT will be tested in worse thermal conditions than in its final position since the temperature of the thermal shield and the conductive heat intercepts will range from $90 \mathrm{~K}$ to $100 \mathrm{~K}$ whereas the real operating conditions will range from $40 \mathrm{~K}$ to $80 \mathrm{~K}$ [3]. The influence of the shield and intercepts temperature on the $4.5 \mathrm{~K}$ load have been already measured with a prototype magnet [4].

The additional important modification to cope with the higher heat loads at $4.5 \mathrm{~K}$ is to increase the two-phase helium flow provided by the centrifugal pump to satisfy the adopted hydraulic stability criteria in the cooling pipes, i.e. to keep a vapor mass fraction at the outlet of the coils less than $10 \%$ and to have a mass flow rate per unit area greater than $4 \mathrm{~g} \cdot \mathrm{s}^{-1} \cdot \mathrm{cm}^{-2}$. An existing centrifugal pump prototype of $600 \mathrm{~g} / \mathrm{s}$ nominal flow, already tested by CEA-Saclay [5], will be re-used at a reduced mass flow of $300 \mathrm{~g} / \mathrm{s}$ to fulfill the hydraulic stability criteria. Since the existing pump cryostat and associated distribution valve box and transfer lines were built for $80 \mathrm{~g} / \mathrm{s}$ required by the BTs, the increased pump flow obliged us to re-design these components and upgrade the associated instrumentation (Fig. 3).

All of this equipment is actually designed and constructed not only to operate the $600 \mathrm{~g} / \mathrm{s}$ pump required for the ECT surface test but also the $1200 \mathrm{~g} / \mathrm{s}$ pumps that have to be tested before their installation in the ATLAS final system.

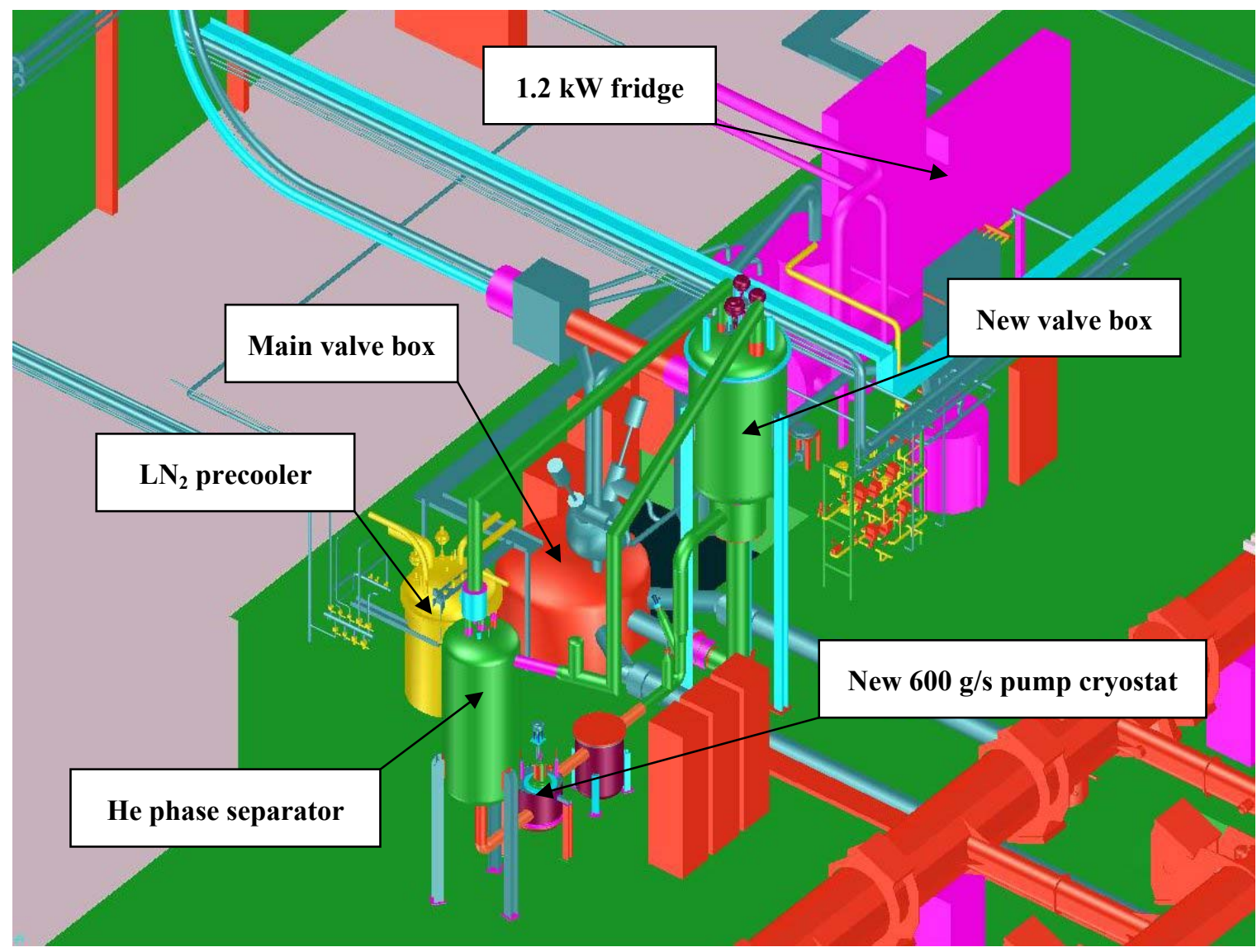

FIGURE 3: Three-dimensional view of the cryogenic equipments required for the ECT tests. 


\section{OPERATING SCENARIOS}

From the cryogenic point of view, the complete test sequence of a single magnet (BT or ECT) consists of three main phases: cool-down from $300 \mathrm{~K}$ to $4.5 \mathrm{~K}$, steady-state operation at $4.5 \mathrm{~K}$ and warm-up back to ambient temperature.

During the first part of the magnet cool-down, i.e. from $300 \mathrm{~K}$ to $100 \mathrm{~K}$, it is very important to avoid excessive thermal stresses induced by a large temperature gradient between two points of the cold mass. A helium forced-flow of maximum $50 \mathrm{~g} / \mathrm{s}$ will be provided by the $\mathrm{LN}_{2}$ precooler which will constantly keep the supplied gas temperature $40 \mathrm{~K}$ lower than the cold mass average temperature.

Once the $100 \mathrm{~K}$ temperature level is reached, there are two different scenarios depending of the magnet type. In the case of a single BT coil, we can switch off the precooler and continue the cooling down to $4.5 \mathrm{~K}$ with solely the $1.2 \mathrm{~kW}$ refrigerator capacity, thus liberating the precooler for cooling a second BT from $300 \mathrm{~K}$ to $100 \mathrm{~K}$. This will allow a considerable reduction of the BT overall test time. On the contrary, if the magnet is an ECT, we have to keep running the precooler which will be connected solely to the thermal shield. The $1.2 \mathrm{~kW}$ refrigerator will then only cope with the $4.5 \mathrm{~K}$ cold mass heat loads and the cooling of the current leads. From $300 \mathrm{~K}$ to $4.5 \mathrm{~K}$, we expect a cooldown time of 3 weeks in the case of a single BT (limited by the temperature gradient) and 6 weeks for an ECT (limited by the available cooling capacity).

The steady-state operation for a BT will also differ from the ECT one. The BT operation at $4.5 \mathrm{~K}$ will not need the $\mathrm{LN}_{2}$ precooler and will require $80 \mathrm{~g} / \mathrm{s}$ of helium forced-flow. The ECT will require $300 \mathrm{~g} / \mathrm{s}$ of helium forced-flow and the precooler on duty all through the test.

Similarly to the cool-down phase, the warm-up is also split into two parts. From $4.5 \mathrm{~K}$ up to $100 \mathrm{~K}$, only direct electrical heating through the windings will be adopted for both BT and ECT. Between $100 \mathrm{~K}$ and $300 \mathrm{~K}$, BTs will continue the warm-up solely via this electrical heating, whereas the complex mechanical structure of the ECT will require an additional forced-flow of gaseous helium to distribute the heat produced electrically and thus smooth the temperature gradients of the cold mass. The warm-up time from $4.5 \mathrm{~K}$ to $300 \mathrm{~K}$ for both BTs and ECTs is similar to the cool-down time.

From the schedule point of view, we have to test first the eight BT coils. Then, after the 8th BT, a short interruption to install the new equipment will be necessary prior to continue the testing of the two ECTs. This intensive test campaign will start with the first BT in January 2004 and should be finished within two years. To accelerate the ATLAS assembly in the cavern, once a BT has been fully tested on the surface, it will be moved underground and mounted on the final structure.

\section{CONCLUSIONS}

The cryogenic test facility for the eight BT coils is now fully operational and ready for final acceptance of these magnets. Concerning the test of the two ECTs that will come after the BTs, we have to build and integrate in the existing test station the $600 \mathrm{~g} / \mathrm{s} \mathrm{pump}$ cryostat and associated equipment. At the end of the two-year continuous test campaign, all the thermo-hydraulic, mechanical, electrical characteristics, including the magnet protection, will be thoroughly verified for every BT and ECT prior to their final underground installation in the ATLAS cavern. 


\section{ACKNOWLEDGEMENT}

Special thanks to E. Coussy who provided a valuable technical assistance for the 3-D design of the ECT test station.

\section{REFERENCES}

1. Haug F., Cambon A., Delruelle N., Orlic J-P., Passardi G. and Tischhauser J., "The CERN cryogenic test facility for the ATLAS Barrel Toroid magnets", in IEEE Transactions on Applied Superconductivity, The 16th International Conference on Magnet Technology, IEEE, March 2000, Vol. 10, pp. 1514-1517.

2. Delruelle N., Dudarev A., Haug F., Mayri C., Orlic J-P., Passardi G., Pirotte O. and Ten Kate H., "First cryogenic testing of the ATLAS superconducting prototype magnets", in IEEE Transactions on Applied Superconductivity, The 17th International Conference on Magnet Technology, IEEE, March 2002, Vol. 12, pp. 1336-1338.

3. Delruelle N., Haug F., Passardi G. and Ten Kate H., "The helium cryogenic system for the ATLAS experiment", in IEEE Transactions on Applied Superconductivity, The 16th International Conference on Magnet Technology, IEEE, March 2000, Vol. 10, pp. 1511-1513.

4. Pengo R., Dolgetta N., Junker S., Passardi G. and Ten Kate H., "Heat load measurements on a large superconducting dipole magnet: an application of a void fraction meter", to be presented at the 18th International Conference on Magnet Technology in October 2003 and published in IEEE Transactions on Applied Superconductivity.

5. Mayri C., Berthier R., Genini L., "Test of a high mass flow rate pump in He I", The 19th International Cryogenic Engineering, Conference Proceedings, July 2002, pp. 75-78. 